\title{
Structure and hardness of cold-rolled nickel after single and multiple electric pulse treatment
}

\author{
I. Sh. Valeev ${ }^{1,2}$, A. Kh. Valeeva ${ }^{\dagger, 1,2}$, R. R. Ilyasov ${ }^{1}$, O. Sh. Sitdikov ${ }^{1}$, M. V. Markushev ${ }^{1}$ \\ †valeevs@mail.ru
}

\author{
${ }^{1}$ Institute for Metals Superplasticity Problems RAS, 39 S. Khalturin St., Ufa, 450001, Russia \\ ${ }^{2}$ Bashkir State University, 32 Zaki Validi St., Ufa, 450076, Russia
}

\begin{abstract}
The structure and hardness of nickel Grade NP2 after rolling at room temperature with a strain of $90 \%$ and subsequent electric pulse treatment with a calculated heating temperature of $550^{\circ} \mathrm{C}$ carried out 1 to 20 times with a pulse duration of $10^{-4} \mathrm{~s}$ and intervals between the pulses of $2 \mathrm{~min}$, were investigated. It has been established that electric pulse treatment activates the static recovery and recrystallization of the heavily deformed structure. After one current pulse, a partially recrystallized, and after 5 pulses, a completely recrystallized fine-grain structure with a grain size of $2-4 \mu \mathrm{m}$ was formed, whose subsequent transformation with number of pulses was accompanied by a slight normal grain growth. It was found that the transformation of the deformation structure of nickel was associated with the intense formation of annealing twins, the fraction of which in the grain boundary spectrum reached $45 \%$ after 5 pulses and further scarcely changed. Such structural changes lead to a decrease in the hardness of the material from 225 to $110 \mathrm{HV}$ followed by subsequent stabilization of its level under processing. The nature of the processes controlling the changes in the structure and hardness of nickel depending on the number of cycles of electric pulse treatment is discussed.
\end{abstract}

Keywords: nickel, electric pulse treatment, structure, hardness, recrystallization.

\section{Introduction}

The effect of electric current on the structure and mechanical properties of metals and alloys has been actively studied for over 50 years and to date a number of significant results have been obtained [1-12]. For example, it has been reliably established that when a metal material is subjected to a straining, the simultaneously applied electric field can significantly change its deformation behavior, in particular, increase the creep rate, reduce the flow stress, and reduce strain hardening. Such a response of the metal is usually associated with a higher mobility of dislocations under the action of an electric current. The physical nature of this effect is associated either with the effect of the interaction of conduction electrons with dislocations, or with the thermal effect that occurs when a current flows through the metal under study.

Most researchers consider the latter to be the main controlling factor, even in treatment based on millisecond current pulses. For instance, in [6-10] it has been stated that the recrystallization processes observed during such an electric pulse treatment (EPT) are caused, first of all, by Joule heating. At the same time, a distinctive feature of EPT compared to conventional annealing in a furnace is a high heating rate (of the order of $10^{-4} \mathrm{~s}$ ) of the processed material, and also a high cooling rate (of about $10^{-1} \mathrm{~s}$ ), which is an important factor determining the parameters of the formed structure. However, despite the fairly large amount of studies, many aspects of the phenomenology and nature of the impact of EPT on the structure and properties of metallic materials are still unclear. In particular, there is no systematic analysis of changes in the structure of a deformed metal during repeated processing.

The purpose of the work is the characterization, using modern experimental techniques, of the microstructure of cold rolled nickel before and after treatment with single and multiple high-density current pulses.

\section{Material and research methods}

Nickel Grade NP2 (99.5\% Ni) was taken as the material for the study. The initial billet was first annealed at $600^{\circ} \mathrm{C}$ for $2 \mathrm{hrs}$, after that it was rolled at room temperature to a total strain of $90 \%$. The subsequent EPT was performed on flat samples $0.4 \mathrm{~mm}$ thick, having a shape similar to samples for mechanical tests with a working part of $3 \times 4 \mathrm{~mm}^{2}$. Samples cut along the rolling direction were fixed in massive copper grips through which current was supplied. With a pulse duration of about $10^{-4} \mathrm{~s}$ and a current frequency of about $10^{4} \mathrm{~Hz}$, the penetration depth in the nickel sample was approximately $0.2 \mathrm{~mm} \mathrm{[3],} \mathrm{which} \mathrm{means} \mathrm{that} \mathrm{for} \mathrm{the} \mathrm{selected}$ thickness of the samples, the skin effect can be neglected and the current distribution over their cross section be considered homogeneous.

It is believed that a material in the case of EPT is heated in accordance with the Joule-Lenz law. Moreover, due to the short exposure time, instrumental recording of the heating temperature of the working part of the sample is extremely 
difficult. Therefore, its value was calculated from the following relation:

$$
\frac{j^{2}}{\sigma_{e}}=\rho c \frac{\partial T}{\partial t}
$$

where $j$ is the current density, $\rho, c, \sigma_{e}$ are the density, heat capacity and electrical conductivity of nickel, respectively. Integrating this equation, we obtained and plotted the dependence of the calculated temperature on the current density $S\left(T_{C}\right)=K_{j}$, where

$$
S\left(T_{C}\right)=\int_{T_{1}}^{T_{C}} \rho c \sigma_{e} d T \text { and } \quad K_{j}=\int_{0}^{\tau} j^{2} d t
$$

$T_{C}$ is the final temperature (to be calculated), $T_{1}$ is the initial temperature of the sample, $\tau$ is the electric pulse duration. For the selected parameters of the current and the size of the samples, the calculated temperature of the heating of the sample was approximately $550^{\circ} \mathrm{C}$. After pulsed heating, the sample rapidly (over a period of about $10^{-1} \mathrm{~s}$ ) cooled down due to the intense heat removal through massive grips. Processing was carried out up to twenty times, while the interval between pulses was at about 2 minutes.

Microstructure of nickel was analyzed in the rolling plane by SEM-EBSD analysis using a TESCAN MIRA 3 LMH microscope and the HKL Channel 5 software package. Diffraction patterns were indicated along 6 Kikuchi lines with a scanning step of no more than $0.5 \mu \mathrm{m}$. A misorientation angle of $15^{\circ}$ was used as a criterion for dividing into low and highangle boundaries (LABs and HABs). The size of the (sub) grains was determined by the equivalent diameter method. The average angle of misorientation of the intercrystalline boundaries $\left(\Theta_{\mathrm{av}}\right)$ and the fraction of high angle boundaries $\left(F_{\mathrm{HABs}}\right)$ in the structure of the material were determined from the distribution diagrams of the misorientations of the boundaries constructed in steps of $1^{\circ}$ (the boundaries with the misorientation $\Theta<2^{\circ}$ were not taken into account). $\Theta_{a v}$ is the "weighted average" value along the misorientation angles in the distribution diagrams, and $F_{\mathrm{HABS}}$ is the result of summing of the fractions of all boundaries with the misorientation angle $\Theta \geq 15^{\circ}$. The fraction of recrystallized grains $\left(F_{\text {rec }}\right)$ was calculated as the ratio of the area of recrystallized grains to the area of all grains.

Microhardness of nickel was determined in the central part of the samples using Vickers digital microhardness tester MVDM 8 "AFFRY" at a load of $0.5 \mathrm{~N}$ and a duration of 10 seconds. At least ten measurements per point were carried out, with an error not exeeding $5 \%$.

\section{Results and discussion}

Typical structure obtained using EBSD analysis and the data on hardness of nickel after rolling are shown in Fig. 1 and Table 1. As one can see, the structure is of a mixed and heavily cold-worked type. Inside the fragments of the initial grains, a developed substructure was formed with crystallites of various shapes having an average size of about $0.3 \mu \mathrm{m}$ and separated mainly by low-angle boundaries.At the same time, there could be found also individual equiaxed dynamically recrystallized grains up to $2-3 \mu \mathrm{m}$ in size surrounded by high-angle boundaries (Fig. 1). That is why the fraction of HABs did not exceed $15 \%$ with an average boundary misorientation angle $\left(\Theta_{\text {av }}\right)$ of about $10^{\circ}$ (Table 1). The metal with this structure showed a microhardness of up to $225 \mathrm{HV}$, which is more than two times higher than the hardness in the state before rolling.

Table 1. Parameters of structure and hardness of cold-rolled nickel before and after electro-pulse treatment.

\begin{tabular}{|c|c|c|c|c|c|c|c|c|}
\hline $\begin{array}{c}\text { Number of } \\
\text { pulses }\end{array}$ & $\begin{array}{c}\text { Grain size, } \\
\mu \mathrm{m}\end{array}$ & $\begin{array}{c}\text { Subgrain size, } \\
\mu \mathrm{m}\end{array}$ & $F_{\text {rec }} \%$ & $F_{\mathrm{HABs}} \%$ & $\begin{array}{c}\Theta_{\mathrm{av}}, \\
\text { degree }\end{array}$ & $F_{\Sigma 3}, \%$ & $F_{\Sigma 9}, \%$ & $\mathrm{HV}$ \\
\hline 0 & 2.0 & 0.3 & 1 & 14 & 10 & 1 & 0 & 228 \\
\hline 1 & 2.9 & 1.2 & 26 & 25 & 14 & 4 & 0 & 158 \\
\hline 5 & 3.5 & - & 96 & 95 & 49 & 42 & 3 & 114 \\
\hline 20 & 3.9 & - & 95 & 95 & 48 & 39 & 4 & 107 \\
\hline
\end{tabular}

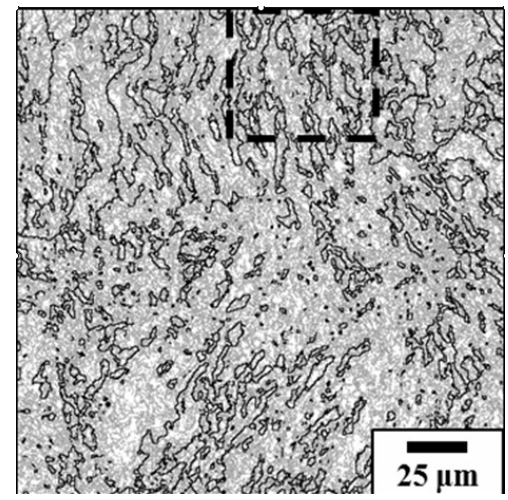

a

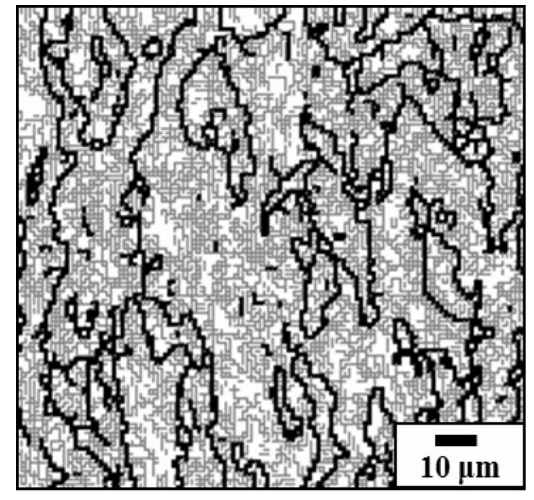

b

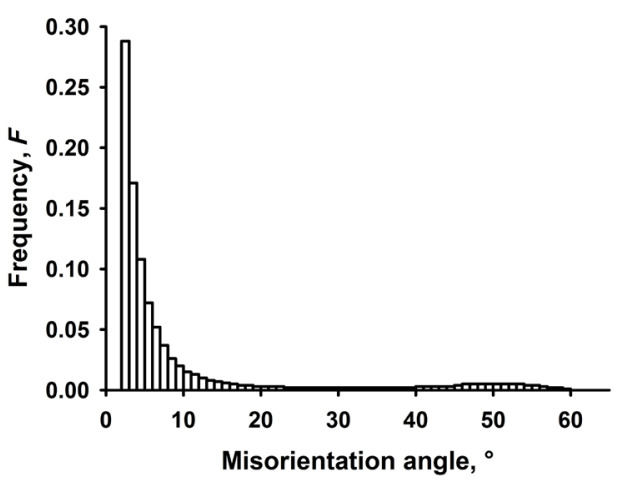

c

Fig. 1. EBSD map of grain boundaries in nickel after rolling (a) and enlarged fragment (highlighted in (a)) (b), as well as the grain boundary spectrum (c) (here and after, low- and high-angle boundaries indicated by grey and black lines, consequently). 
After a single EPT, a decrease in microhardness by $60 \mathrm{HV}$ was observed, which indicated the intense relaxation of accumulated internal stresses. Judging by the map in Fig. $2 \mathrm{a}$, in addition to static recovery, static recrystallization processes also began to develop in the deformed material, the main of which were primary recrystallization and abnormal growth of individual new small grains. As a result, a partially recrystallized structure with an average size and fraction of recrystallized grains of $2.9 \mu \mathrm{m}$ and $26 \%$, respectively, inside large non-crystallized regions of about $100 \mu \mathrm{m}$ in size was formed in the material. In this case, the characteristic arrangement of recrystallized grains in the form of chains (lines) and their periodicity testified to the fact that the places of the predominant nucleation of new grains were the boundaries of the initial grains and adjacent areas, and the unrecrystallized regions were fragments of the initial grains. At the same time, after the EPT, the fraction of HABs and the average angle of misorientation of the boundaries significantly increased (Table 1). It is noteworthy that this is largely due to the formation of annealing twins ( 23 ) (Figs. 3 and 4). This fact was also observed in [12-17] during furnace annealing of cold rolled nickel at various temperatures and times.

After 5 pulses of EPT, a completely recrystallized homogeneous fine-grained structure with a grain size of $3.5 \mu \mathrm{m}$ was formed in nickel (Fig. $2 \mathrm{~b}$ ). Such a structure was characterized by the almost complete absence of low-angle boundaries (Fig. 3) and the average angle of misorientation

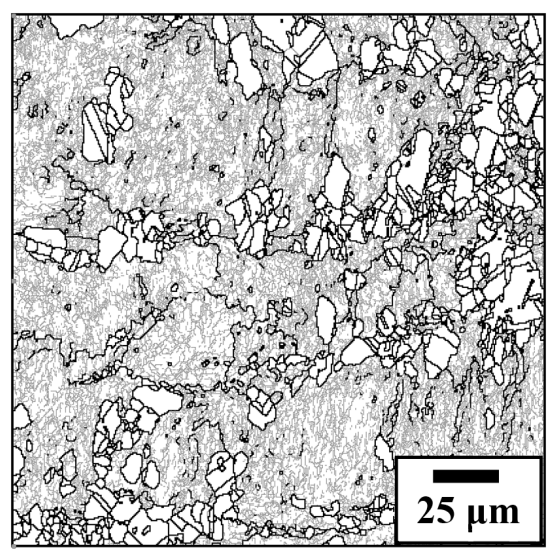

a

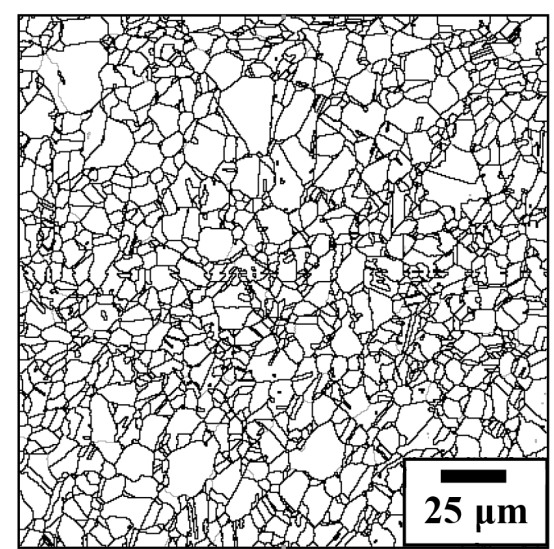

$\mathrm{b}$

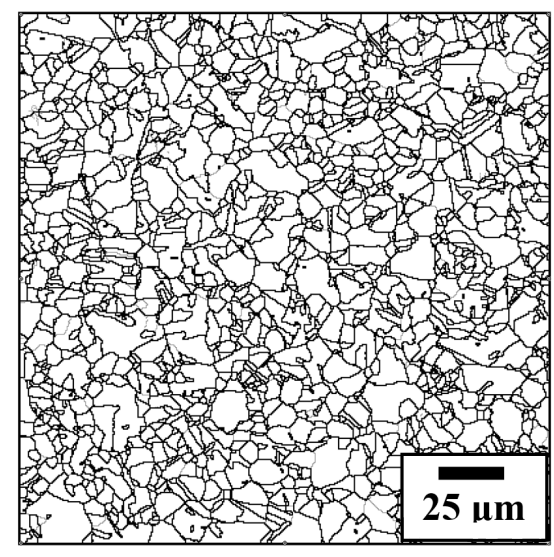

C

Fig. 2. EBSD grain boundary maps of cold-rolled and 1 (a), 5 (b) and 20 pulse (c) treated nickel.

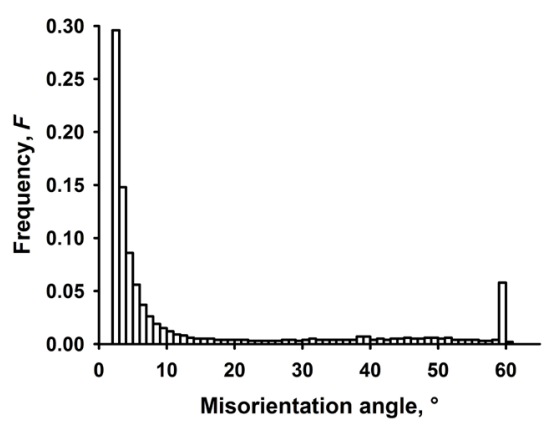

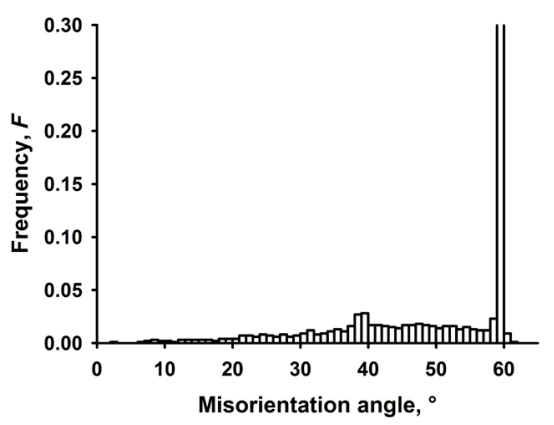

b

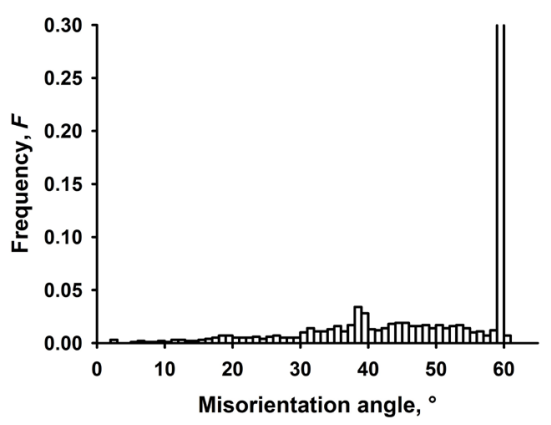

c

Fig. 3. Grain boundary spectrums in nickel after 1 (a), 5 (b) and 20 pulses (c) treatment.

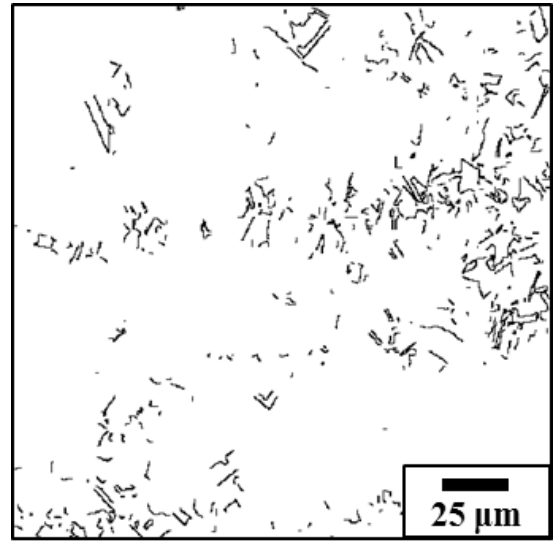

a

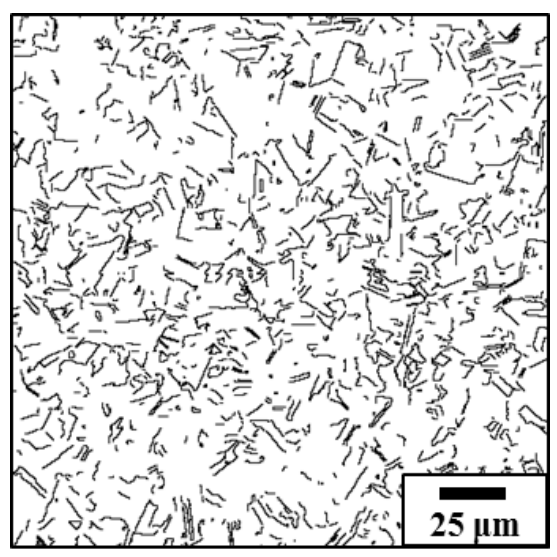

b

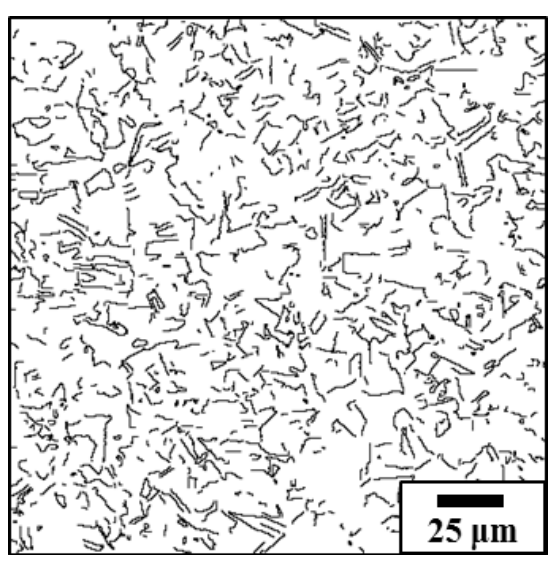

C

Fig. 4. EBSD twin ( $\Sigma 3$ ) maps in cold-rolled nickel after 1 (a), 5 (b) and 20 pulses (c) treatment. 
of the boundaries, which reached almost $50^{\circ}$ (Table 1). At the same time, two peaks corresponding to the special boundaries $\Sigma 3$ and $\Sigma 9$ were observed (Fig. $3 \mathrm{~b}$ ). The fraction of such boundaries was 42 and $3 \%$, respectively (Table 1), which once again indicates the dominance of recrystallization processes, accompanied by the formation of annealing twins.

A further increase in the number of pulses to 20 led only to insignificant normal grain growth up to about $4 \mu \mathrm{m}$ without a noticeable change in other structure parameters. In this case, the hardness practically did not change and stabilized at the level of about $110 \mathrm{HV}$ (Table 1).

One can conclude from the qualitative and quantitative characteristics of the recrystallized structures of nickel obtained after furnace annealing [12-17] and after EPT in this study that in both cases annealing twins formed mainly inside the largest abnormally growing grains, i.e. the mechanism of the formation of twins was due to the same process - the growth of recrystallized grains. This indicates the unity of the physical nature of the structural changes that occur during the annealing of highly deformed nickel, which does not depend on the parameters and method of its implementation.

The model describing the sequence of the formation of twins during furnace annealing was proposed in [13]. It starts from stacking fault occurrence at the migrating boundary, which then leads to the generation of coherent twin boundaries and their subsequent propagation from triple junctions into the growing grain. It can be stated that the results of this work are in favor of this model, and also confirm the point of view that the main factor controlling the structural transformations during nickel EPT is the release of Joule heat. Significant difference of the processing by current pulses is the formation of a finer and more homogeneous structure containing a network of grain boundaries, almost half consisting of special boundaries. An advantage of such a structure is its high stability due to low internal energy and mobility of special boundaries [11].

\section{Conclusion}

It has been shown that the phenomenology and nature of the processes of transformation of the structure of coldrolled nickel during electropulse treatment and conventional furnace heating are the same. In both cases, the processes of static recovery and recrystallization proceed, accompanied by the formation of annealing twins in the recrystallized regions. At that, repeated exposure to current pulses of the selected power, in addition to completion of recrystallization processes, leads to an increase in the proportion of twin boundaries to $45 \%$, which contributes to the formation of a more uniform and fine grained structure. Such structural transformations are accompanied by a noticeable softening of nickel: as the recrystallization processes are completed, the microhardness decreases almost two times (to $110 \mathrm{HV}$ ) and then remains practically unchanged.

Acknowledgements. The work was supported by the RFBR under grant No. 19-08-00953A. The work was performed using the facilities of the shared services center "Structural and Physical-Mechanical Studies of Materials" at the Institute for Metals Superplasticity Problems of Russian Academy of Sciences.

\section{References}

1. H. Conrad, N. Karam, S. Mannan. Scr. Metall. 17 (3), 411 (1983). Crossref

2. H. Conrad, A. F. Sprecher, W. D. Cao, X. P. Lu. JOM. 42, 28 (1990). Crossref

3. H. Knoepfel. Pulsed High Magnetic Fields. Amsterdam, North-Holland Publishing Company (1970) 392 p.

4. Yu.V. Baranov, O.A. Troitsky, Yu.S. Avramov, A.D. Shlyapin. Physical fundamentals of electropulse and electroplastic treatments and new materials. Moscow, MSIU (2001) 844 p. (in Russian)

5. W. Jin, J. Fan, H. Zhang, Y. Liu, H. Dong, B.Xu. J. All. Com. 646, 1 (2015). Crossref

6. I. Sh. Valeev, N. P. Barykin, V. G. Trifonov, A. Kh. Valeeva. J. Mater. Eng. Perform. 14, 236 (2005). Crossref

7. T. Konkova, I. Valeev, S. Mironov, A. Korznikov, M. Myshlyaev, S. Lee Semiatin. J. Mater. Res. 29, 2727 (2014). Crossref

8. I. Sh. Valeev. Lett. on Mater. 3 (3), 236 (2013). (in Russian) $\underline{\text { Crossref }}$

9. E. V. Avtokratova, R. R. Ilyasov, I. S. Valeev, O. S. Sitdikov, M. V. Markushev. Lett. on Mater. 1 (4), 194 (2011). (in Russian) Crossref

10. I. Sh. Valeev, A. Kh. Valeeva, A.Kh. Akhunova. Basic Probl. of Mat. Sci. 12 (2), 214 (2015). (in Russian)

11. F.J. Humphreys, M. Hatherly. Recrystallization and related annealing phenomena. 2nd ed. Elsevier (2004) 605 p. Crossref

12. M. Booth, V. Randle, G. Owen. J. Micros. 217, 162 (2005). Crossref

13. X.-M. Chen, Y.C. Lin, Fan Wu. J. All. Com. 724. 198 (2017). Crossref

14. V. Randle, P. R. Rios and Y. Hu. Scr. Mater. 58, 130 (2008). Crossref

15. J. L. Bair, S. L. Hatch, D. P. Field. Scr. Mater. 81, 52 (2014). Crossref

16. Y. Jin, B. Lin, M. Bernacki, G.S. Rohrer, A. D. Rollett, N. Bozzolo. Mat. Sci. Eng. A. 597, 295 (2014). Crossref

17. X. P. Chen, L. F. Li, H. F. Sun, L. X. Wang, Q. Liu. Mat. Sci. Eng. A. 622, 108 (2015). Crossref 